\title{
$\mu$-Sensitivities as an Aid for Robust Identification
}

\author{
R. D. Braatz and M. Morari* \\ Chemical Engineering 210-41 \\ California Institute of Technology \\ Pasadena, CA 91125
}

\section{Abstract}

Identification for a model for robust control design is more complicated than for the standard linear system transfer function model-the structure of the uncertainty as well as bounds on its size must be determined. It is especially unclear as to which parts of the system should be better modeled to improve robust performance. This paper addresses this question through some new tools, the $\mu$-sensitivities.

\section{Introduction}

\subsection{Robust Performance}

The goal of any controller design is that the overall system is stable and satisfies some minimum performance requirements. These requirements should be satisfied at least when the controller is applied to the nominal plant, that is, we require nominal stability and nominal performance.

In practice the real plant $G_{p}$ is not equal to the model $G$. The term "robust" is used to indicate that some property holds for a set $\Pi$ of possible plants $G_{p}$ as defined by the uncertainty description. In particular, by robust performance we mean that the performance requirements are satisfied for all $G_{p} \in \Pi$. Performance is commonly defined in robust control theory using the $H_{\infty}$-norm.

Definition 2.1 The closed loop system exhibits nominal performance if

$$
\bar{\sigma}(\Sigma) \leq 1, \quad \forall \omega .
$$

Definition 2.2 The closed loop system exhibits robust performance if

$$
\bar{\sigma}\left(\Sigma_{p}\right) \leq 1, \quad \forall \omega, \quad \forall G_{p} \in \Pi .
$$

For example, a simple choice for $\Sigma$ could be the weighted sensitivity

$$
\begin{array}{ll}
\Sigma=W_{1} S W_{2}, & S=(I+G C)^{-1} \\
\Sigma_{p}=W_{1} S_{p} W_{2}, & S_{p}=\left(I+G_{p} C\right)^{-1} .
\end{array}
$$

The input weight $W_{2}$ is often equal to the disturbance model. The output weight $W_{1}$ is used to specify the

\footnotetext{
- Correspondence should be sent to: phone (818)356-4186, fax (818) 568-8743, e-mail mm Gimc.caltech.edu
}

frequency range over which the sensitivity function should be small and to weight each output according to its importance. $C$ is the transfer function of the controller.

Doyle [3] derived the structured singular value, $\mu$, to test for robust performance. To use $\mu$ we must model the uncertainty (the set $\Pi$ of possible plants $G_{p}$ ) as norm bounded perturbations $\left(\Delta_{i}\right)$ on the nominal system. Through weights each perturbation is normalized to be of size one:

$$
\bar{\sigma}\left(\Delta_{i}\right) \leq 1, \quad \forall \omega .
$$

The perturbations, which may occur at different locations in the system, are collected in the diagonal matrix $\Delta_{U}$ (the $U$ denotes uncertainty)

$$
\Delta_{U}=\operatorname{diag}\left\{\Delta_{1}, \cdots, \Delta_{n}\right\}
$$

and the system is arranged to match the structure in Figure 1. The interconnection matrix $M$ in Figure 1 is determined by the nominal model $(G)$, the size and nature of the uncertainty, the performance specifications, and the controller $(C)$. The definition of $\mu$ is:

Definition 2.3 Let $M$ be a square complex matrix. $\mu(M)$ is defined such that $\mu^{-1}(M)$ is equal to the smallest $\bar{\sigma}(\Delta)$ needed to make $(I+\Delta M)$ singular, i.e.

$\mu^{-1}(M)=\min _{\delta}\{\delta: \operatorname{det}(I+\Delta M)=0$ for some $\Delta, \bar{\sigma}(\Delta)<\delta\}$.

For Figure 1, the robust performance condition (2.2) becomes [3]:

Theorem 2.4 The closed loop system exhibits robust performance if and only if the closed loop system is nominally stable and

$$
\mu_{R P}(M) \equiv \sup _{\omega} \mu(M)<1 .
$$

$\mu(M)$ depends on both the elements in the matrix $M$ and the structure of the perturbation matrix $\Delta=$ $\operatorname{diag}\left\{\Delta_{U}, \Delta_{P}\right\} . \Delta_{P}$ is a full square matrix with dimension equal to the number of outputs (the subscript $P$ denotes performance). Note that the issue of robust stability is simply a special case of robust performance. It is also a key idea that $\mu$ is a general analysis tool for determining robust performance. 


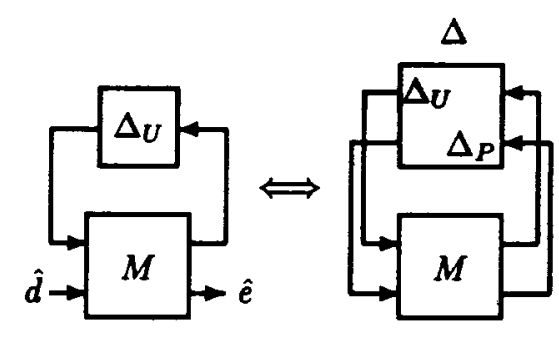

Figure 1: Robust Performance and the $M-\Delta$ block structure

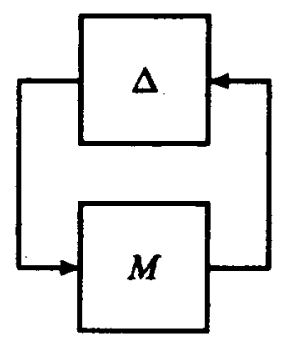

Figure 2: The $M-\Delta$ block structure

Any system with uncertainty adequately modeled as in (2.4) can be put into $M-\Delta$ form, and robust performance can be tested using (2.6). Standard programs calculate the $M$ and $\Delta[1]$, given the transfer functions describing the system components and the location of the uncertainty blocks $\Delta_{i}$. Besides meeting some specified performance requirements, often the controller is constrained as well. What this means is that the control designer may want to use PID controllers only, or a decentralized controller, or to use a controller of limited order. The controller design procedure described below allows constrained controllers.

\subsection{Designing for Robust Performance}

A controller design method using $\mu$-analysis looks like this:

1. Determine model of plant (including uncertainty model).

2. Design controller using whatever method you choose (e.g. $\mu$-synthesis [4], decentralized controller design using independent designs [17], $\mu$ optimal single-loop PID controller design [11]).

3. Test for robust performance using $\mu$

- If $\mu_{R P}<1$, then the controller meets robust performance and the design is complete.

- If $\mu_{R P}>1$ then the controller does not meet robust performance.

If $\mu_{R P}>1$, then either the performance specifications must be weakened or the model must be improved. If the performance specifications are set, then a more accurate model must be determined. It is currently not clear to the control designer to what part of the model should attention be focused for improving the model. This is important because we would like to meet the performance requirements with a minimum amount of modeling effort. After all, modeling is the most time-consuming, expensive, and difficult step in controller design. We do not want the control engineer wasting effort improving the model where the performance is only marginally improved.

In robust control language, the above question is: where should uncertainty be decreased to have the largest effect in improving robust performance? This paper addresses this question through some new functions, the $\mu$-sensitivities. The $\mu$-sensitivities aid the control designer in deciding which uncertainties should be focused on for meeting robust performance requirements.

\section{Definition of $\mu$-Sensitivities}

\subsection{Definition}

Let us look once again at Figure 1. Partition

$$
M=\left[\begin{array}{ll}
M_{11} & M_{12} \\
M_{21} & M_{22}
\end{array}\right]
$$

to be compatible with $\Delta=\operatorname{diag}\left\{\Delta_{U}, \Delta_{P}\right\}$. We want to measure the sensitivity of the robust performance measure $\mu_{R P}(M)$ with respect to the size of the individual uncertainties $\Delta_{i}$.

To study this problem, multiply each $\Delta_{i} \in \Delta_{U}$ by $\alpha_{i}$, where each $\alpha_{i}$ is real and nominally 1 . Then

$$
\operatorname{diag}\left\{\alpha_{1} \Delta_{1}, \cdots, \alpha_{n} \Delta_{n}\right\}=\alpha^{1 / 2} \Delta_{U} \alpha^{1 / 2},
$$

where

$$
\alpha=\operatorname{diag}\left\{\alpha_{1} I_{r_{1}}, \cdots, \alpha_{n} I_{r_{n}}\right\},
$$

and $r_{i}$ is the dimension of the $i$ th uncertainty $\Delta_{i}$.

Absorb the $\alpha^{1 / 2}$ 's into $M$ to give

$$
M(\alpha)=\left[\begin{array}{cc}
\alpha M_{11} & \alpha^{1 / 2} M_{12} \\
\alpha^{1 / 2} M_{21} & M_{22}
\end{array}\right] .
$$

Define $M\left(\alpha_{i}\right)$ to be equal to $M(\alpha)$ for $\alpha$ such that $\alpha_{j}=1, \forall j \neq i$. The following is the definition of the $i$ th $\mu$-sensitivity.

$\partial \mu_{i} \equiv\left[\left.\lim _{\Delta \alpha_{i} \rightarrow 0+} \frac{\mu_{R P}\left(M\left(\alpha_{i}\right)\right)-\mu_{R P}\left(M\left(\alpha_{i}-\Delta \alpha_{i}\right)\right)}{\Delta \alpha_{i}}\right|_{\alpha=1}\right.$

Recall that each $\alpha_{i}$ is nominally one.

Remark 3.1 Because the $\Delta_{i}$ are normalized to have maximum norm 1, the $\mu$-sensitivities are defined on a "relative basis". In other words, each $\mu$-sensitivity is measured based as percentage reduction of the associated uncertainty block, not in the absolute reduction in the size of each uncertainty in the system.

Remark 3.2 Any pertirbation $\Delta_{i} \in \Delta_{U}$ can be of any form (i.e. real or complex, repeated scalar or fullblock, or repeated blocks). Withaut loss of generality, we can consider only square $\left.\Delta_{i}[1\}\right]$. 
Remark 3.3 We cosld have defined the matrix $M(\alpha)$ to have the form

$$
M^{\prime}=\left[\begin{array}{cc}
\alpha M_{11} & \alpha M_{12} \\
M_{21} & M_{22}
\end{array}\right]
$$

or,

$$
M^{\prime \prime}=\left[\begin{array}{ll}
\alpha M_{11} & M_{12} \\
\alpha M_{21} & M_{22}
\end{array}\right],
$$

since

$$
\mu_{R P}(M(\alpha))=\mu_{R P}\left(M^{\prime}(\alpha)\right)=\mu_{R P}\left(M^{\prime \prime}(\alpha)\right)
$$

The form in (9.10) was chosen for computational reasons as discussed in Appendix $B$.

Remark 3.4 It is easy to show that $\mu_{R P}\left(M\left(\alpha_{i}\right)\right)$ is a nondecreasing function of $\alpha_{i}$. This implies that $\mu$ sensitivities are always non-negative.

\subsection{Existence}

$\mu_{R P}(M(\alpha))$ is continuous in $\alpha$ (this follows directly from [15] and that we have a complex performance block). Although the slope of $\mu_{R P}\left(M\left(\alpha_{i}\right)\right)$ as a function of $\alpha_{i}$ can be discontinuous, the one-sided derivatives defining the $\mu$-sensitivities will exist and be finite except for a set of measure zero. We will use the following lemma from [9] to prove that not only is the one-sided derivative well-defined, the full derivative is well-defined (and equal to the one-sided derivative) almost everywhere.

Lemma 3.5 A monotonic function defined on an interval has a finite derivative almost everywhere on the interval.

The next theorem then directly follows.

Theorem 3.6 The $\mu$-sensitivities are well-defined, and equal to the corresponding full derivatives, almost everywhere on any interval.

\section{Computational Issues}

The exact calculation of $\mu$ for general real and complex $\Delta_{i}$ is not currently available.

Two main approaches are taken to computing $\mu$. The first approach calculates $\mu$ exactly, but involves an exponential growth in computation as a function of the parameters. The best of these methods are rapid when the number of $\Delta_{i}$ blocks is small $(<-10)[16]$.

The second approach is more computationally attractive, but can be inexact. Computationally inexpensive upper and lower bounds are calculated (see Appendix A for more details); if the bounds are equal, then an exact value for $\mu$ has been found. For strictly complex $\Delta$, the upper and lower bounds are almost always within a percent or so for real problems [14], thus for engineering purposes, $\mu$ never has to be exactly calculated. The upper and lower bounds also appear to be tight for the mixed uncertainty case $[19,20,7]$.
Tight upper and lower bounds allow us to analyze the performance (and stability) using only the upper, or lower bound, instead of the exact value for $\mu$. The efficient computation of the derivatives will be discussed with respect to $\alpha_{i}$, using the upper and lower bounds in place of the exact value for $\mu$. This possibly could give values different from the exact $\mu$ sensitivities, but as long as our analysis is with respect to the given upper or lower bound, this is not important.

Inexact- $\mu$ calculation procedures are iterative $[3,6$, $14,7,19]$. To numerically compute the derivative, just use the last iteration from the inexact- $\mu$ calculation for $M\left(\alpha_{i}\right)(=M)$ as the first iteration for the inexact- $\mu$ calculation for $M\left(\alpha_{i}-\Delta \alpha_{i}\right)$. The inexact- $\mu$ calculation for $M\left(\alpha_{i}-\Delta \alpha_{i}\right)$ then converges in only a couple of iterations. Since inexact- $\mu$ has already been calculated for $M\left(\alpha_{i}\right)$ (with $\alpha_{i}=1$ ), each numerical calculation of a $\mu$-sensitivity requires only a couple of iterations.

One difficulty that can arise when using the lower bound iterative algorithms $[14,6,19]$, though unlikely for $\Delta \alpha_{i}$ small, is that the procedure can converge to different local extrema (maxima). To avoid this, it is recommended to use the upper bound calculation procedures. The computation of the upper bound is convex, so the only extremum (a minimum) is global $[3,14]$.

We see that the $\mu$-sensitivities can be approximated easily. The calculation requires little more computational effort than that needed to approximate $\mu(M)$.

Remark 4.1 The frequency domain calculation to find $\mu_{R P}$ can be transformed to a $\mu$ calculation involving larger $M$ and $\Delta$ matrices [5]. ${ }^{1} A$ bilinear transformation converts the continuous-time $\mu_{R P}$ problem to a discrete-time $\mu_{R P}$ problem, where the frequency variable is treated as another $\Delta$ block to give a larger $\mu$ problem.

This conversion should be done for the calculation of the $\mu$-sensitivities for two reasons. First, the transformation will allow a faster and simpler calculation for $\mu_{R P}$, since one (though larger) $\mu$ calculation replaces the numerous $\mu$ calculations required in an accurate frequency search needed for derivative calculation. Second, problems which have two or more similarly sized peaks in the $\mu$ versus frequency plot give difficulties in numerically calculating the derivetive defining the $\mu$-sensitivities. Two similarly sized peaks often occur, for example, when the IMC design method is used.

\section{Generalized Design Proce- dure Using $\mu$-Sensitivities}

We will now focus on a general procedure for robust controller design. We will define the plant as the system to be controlled. Components of the plant refer to actuators, sensors, and the physical processes making up the plant. We begin our identification procedure with a nominal model and a broad uncertainty description-such a broad description takes much less effort to generate than a nominal model

\footnotetext{
${ }^{1}$ The larger $\mu$ problem is called skewed- $\mu$, and is calculated by the same methods as $\mu$ [18].
} 
with a tight uncertainty description. We then design a controller and check robust performance. If robust performance is satisfied then the design is complete. If robust performance is not satisfied, our design procedure must then point out which components of the plant should be better identified-this step becomes particularly important for multivariable plants with a large number of components with associated uncertainties. We should not re-identify all components of the plant more accurately, because the uncertainty of some components of the plant may have little effect on robust performance.

The $\mu$-sensitivities measure the sensitivities of the robust performance measure $\mu$ with respect to the magnitude of the individual uncertainties. When the $\mu$-sensitivity corresponding to a particular uncertainty is large, then it is important to diminish that uncertainty for improved robust performance. The control designer should then consider re-identifying (to decrease the size of the uncertainty) the component of the plant corresponding to this uncertainty. When a $\mu$-вensitivity is small, then the corresponding uncertainty is unimportant.

However, re-identifying plant components based strietly on which $\mu$-sensitivities are the largest is naive. Some plant components are inherently harder to identify than others. Some uncertainties are inherently more difficult to model or identify-this is especially true of uncertainties resulting from the ad hoc covering of slowly time-varying parameter variations with linear time-invariant uncertainty, or when no "true" model parameters exist because of simplifying assumptions on the structure of the plant. The control designer must weigh the importance of the uncertainties as suggested by the $\mu$-sensitivities with the difficulty in re-identifying each uncertainty.

Keeping these ideas in mind, we have the following new robust controller design method:

1. Determine model of plant, including a broad uncertainty description

2. Design controller using whatever method you choose (i.e. $\mu$-synthesis, decentralized controller using independent designs, PID controllers)

3. Test for robust performance using $\mu$

- If $\mu_{R P}<1$, then the controller meets robust performance and the design is complete.

- If $\mu_{R P}>1$, then the controller does not meet robust performance. Calculate the $\mu$ sensitivities. Re-identify components of the plant corresponding to large $\mu$-sensitivities and low identification effort. Repeat steps 2 and 3 until design is completed.

Remark 5.1 The ith $\mu$-sensitivity is the derivative of the robust performance measure $\mu_{R P}$ with respect to the magnitude of the ith uncertainty block. In some situations, it makes sense to take the derivative of $\mu_{R P}$ with respect to the magnitude of several ancertainty blocks. This would happen when the plant has several components that have the same ancertainty description, though the ancertainties are independent of each other. This would arise, for example, for uncertainty associated with equivalent, independent actuators. Modifying the definition of the $\mu$-sensitivities is simple.
Remark 5.2 If, at an iteration in the design procedure, the uncertainty is modeled to be of a different form (for example, if a plant component that was originally modeled as parameter uncertainty is modeled as nonparametric uncertainty), then the $M$ and $\Delta$ will change, and the design procedure continues.

\section{Example: Cross-Directional Control in Paper Manufac-}

\section{turing}

\subsection{Description of System}

The description of the problem can be found in [10].

The cross-directional (CD) control problem in paper manufacturing is aimed at maintenance of flat profiles of paper sheet properties across the paper machine. One important sheet property, for example, is the paper weight per unit area. Variations in CD paper weight per unit area can result in paper that will not lie flat. Successful control of CD paper sheet properties can mean significant reductions in raw material consumption. Minimal variation in CD sheet properties enables the production of thinner paper closer to the target caliper. The process model used to describe the system includes three factors always present in CD control problems: actuator dynamics, interactions, and time-delay. All of these components of the system have significant uncertainty associated with them.

$p_{a}(s)$ will represent the model for the actuator, $p_{d}(s)$ will represent the time delay, and $P_{C D}$ is a full transfer function matrix describing the interactions. A model for a $20 \times 20$ plant, assuming symmetric responding actuators and interactions involving only nearest two sensors, is $P_{C D}(s)=p_{a}(s) p_{d}(s) P_{C D}=$

$$
\frac{k_{a} e^{-0 .}}{\tau_{a} s+1} \underbrace{\left(\begin{array}{cccccc}
p_{1} & p_{2} & p_{3} & 0 & \cdots & 0 \\
p_{2} & p_{1} & p_{2} & \ddots & \ddots & \vdots \\
p_{3} & p_{2} & \ddots & \ddots & \ddots & 0 \\
0 & \ddots & \ddots & \ddots & p_{2} & p_{3} \\
\vdots & \ddots & \ddots & p_{2} & p_{1} & p_{2} \\
0 & \cdots & 0 & p_{3} & p_{2} & p_{1}
\end{array}\right)}_{20 \times 20}
$$

We will describe the uncertainty as parametric uncertainty in $k_{a}, \tau_{a}, \theta, p_{2}$, and $p_{3}$, i.e. each parameter is allowed to vary between upper and lower bounds independent of the other real parameters.

Nominal values for $p_{1}, p_{2}$, and $p_{3}$ are taken from actual process data from [2]. The following ranges represent a fairly broad uncertainty description:

$$
p_{1}=1.0 ; p_{2} \in(0.1,0.3) ; p_{3} \in(-0.15,0.0) \text {. }
$$

Actuator dynamics and time-delay vary substantially from machine to machine; we will start our design 
procedure with the following broad ranges for $k_{a}, \tau_{a}$, and $\theta$ :

$$
k_{a} \in(0.5,1.5) ; \quad \theta \in(0.5,1.5) ; \quad \tau_{a} \in(0.5,1.5) .
$$

It is hoped that it is fairly simple to guess the time constant, steady-state gain; and time delay by a factor of two. A tighter uncertainty description would require some identification effort.

\subsection{Choice of Performance Weight}

Our performance objective is to achieve a bandwidth of at least $\frac{1}{4}$ radian per second, under model uncertainty. A performance weight $w(s)$ that assures this is given by:

$$
w(s)=\frac{4 s+1}{8 s}
$$

\subsection{Controller Design Procedure}

A decentralized controller is designed for flexibility in operation, simplicity of design, and operator acceptance. Since the plant is almost diagonal, it is reasonable to use a single loop Internal Model Control (IMC) [13] controller multiplied by the identity matrix. The IMC controller is $q(s)=\tilde{q}(s) f(s)$, where $\tilde{q}(s)$ is $H_{2}$ optimal for step disturbances for $\frac{k_{0} e^{-0}}{r_{s} s+1}$ detuned somewhat to take into account the effect of interactions (the design procedure is discussed in detail in [10]), and $f(s)$ is a robustness filter with time constant $\lambda$. Varying $\lambda$ gives a transparent tradeoff between performance and robustness. For our problem, $\lambda$ is chosen to minimize $\mu_{R P}$.

For our system,

$$
\begin{gathered}
\tilde{q}(s)=\frac{s+1}{0.85} \\
f(s)=\frac{1}{\lambda s+1}
\end{gathered}
$$

The standard feedback controller $c(s)$ (a Smith Predictor) is given by:

$$
c(s)=\frac{1}{0.85} \frac{s+1}{\lambda s+1-e^{-s}} .
$$

\subsection{General Robust Design Procedure}

The first step is done-we have a model with a broad uncertainty description. A controller is designed according to (6.21). The $\lambda$ minimizing $\mu_{R P}$ was 2.34 and the minimized $\mu_{R P}$ was 6.0. Table 1 gives the $\mu$-sensitivities $\left(\partial \mu_{i}\right)$ and the corresponding parameter ranges. Also given are the controller-varying $\mu$ sensitivities $\partial \mu_{i}^{*}$ - these are defined by

$$
\begin{aligned}
& \partial \mu_{i}^{v} \equiv\left[\operatorname { l i m } _ { \Delta \alpha _ { i } \rightarrow 0 + } \frac { 1 } { \Delta \alpha _ { i } } \left(\min _{\lambda} \mu_{R P}\left(M\left(\alpha_{i}\right)\right)-\right.\right. \\
&\left.\min _{\bar{\lambda}} \mu_{R P}\left(M\left(\alpha_{i}-\Delta \alpha_{i}\right)\right)\right)\left.\right|_{\alpha=1}
\end{aligned}
$$

The $\partial \mu_{i}^{\nu}$ are more time-consuming to calculate (requiring an additional controller design for each derivative calculation), but can be argued to be a closer

\begin{tabular}{|l|l|r|r|}
\hline$\mu=6.0$ & & & \\
$\lambda=2.34$ & Range & $\partial \mu_{i}$ & $\partial \mu_{i}^{v}$ \\
\hline$\theta$ & $0.5-1.5$ & 0 & 2 \\
$k$ & $0.5-1.5$ & 12 & 14 \\
$\tau_{a}$ & $0.5-1.5$ & 0 & 2 \\
$p_{2}$ & $0.1-0.3$ & 76 & 94 \\
$p_{3}$ & $-0.15-0.0$ & 75 & 92 \\
\hline
\end{tabular}

Table 1: First Iteration in Design Procedure

\begin{tabular}{|l|l|r|r|}
\hline$\mu=1.8$ & & & \\
$\lambda=2.50$ & Range & $\partial \mu_{i}$ & $\partial \mu_{i}^{v}$ \\
\hline$\theta$ & $0.5-1.5$ & 0 & 0.5 \\
$k$ & $0.5-1.5$ & 2.9 & 3.3 \\
$\tau_{a}$ & $0.5-1.5$ & 0 & 0.4 \\
$p_{2}$ & $0.1-0.2$ & 6.7 & 8.0 \\
$p_{3}$ & $-0.1--0.05$ & 6.5 & 7.8 \\
\hline
\end{tabular}

Table 2: Second Iteration in Design Procedure

measure of the design-relevant sensitivity of the robust performance to the uncertainties. We see that by far the most important uncertainties are associated with the interactions $p_{2}$ and $p_{3}$.

For the CD response system, the interactions can be identified independent of the other plant parameters $\left(k, \theta\right.$, and $\left.\tau_{a}\right)$. A re-identification was "simulated" by decreasing the allowable ranges for $p_{2}$ and $p_{3}$ to $(0.1,0.2)$ and $(-0.1,-0.05)$, respectively. A controller was designed, with the relevant results in Table 2. We see that the most important uncertainties are still associated with the interactions. For this example, we will assume that better identifying $p_{2}$ and $p_{3}$ would require a large amount of effort, at least much more effort than identifying the actuator gain $k$ better. We can see that the uncertainty in $k$ is almost as important as interaction uncertainty (within a factor of two), so we will identify $k$ more accurately. This is "simulated" by decreasing the range of $k$ to $(0.9,1.1)$.

$A$ controller was designed and it was found that $\mu_{R P}=1.0$ for $\lambda=2.64$. The design procedure is complete.

\subsection{Discussion}

The example problem shows the utility of the proposed robust design procedure. For the chosen controller design method, the $\mu$-sensitivities implied that robust performance does not depend strongly on uncertainty in time delay and time constant. Also implied was that interaction uncertainties are by far the most important uncertainties. This agrees with real CD control; McFarlin [12] cites ignorance of interac- 
tion uncertainty as a probable cause of instability in CD response control systems.

We see, for this example, that the extra effort in calculating the controller-varying $\boldsymbol{\mu}$-sensitivities is wasted. Though $\partial \mu_{i} \neq \partial \mu_{i}^{v}$, the trends are still the same. The design procedure uses the $\partial \mu_{i}$ qualitatively instead of quantitatively, so the design procedure is unaffected by which values are used.

\section{Conclusions and Further Re- search}

For identification of a robust control model, the structure of the uncertainty as well as bounds on its size must be determined. It was pointed out that it was especially unclear as to which parts of the system should be better modeled to improve robust performance. A new tool, the $\mu$-sensitivity, was defined in an attempt to address this problem. Computational issues were addressed, and an extensive example showed the utility of $\mu$-sensitivities as an aid in the identification of models required for robust control.

The limitation of $\mu$-sensitivities as an analysis tool is the same as for other (local) sensitivity analyses: no guarantees are available. However, since the $\mu$ sensitivities are used only to focus attention to what parts of the system are important-robust performance is still guaranteed when the design procedure ends with $\mu_{R P}<1$-no guarantees are necessary. The $\mu$-sensitivities complement the information obtained by the structured singular value, with minimal added computation.

This paper can be interpreted as an extension of [8]. The above paragraph applied to the work in [8] also, and is lifted from that paper.

The $\mu$-sensitivity is a specialized one-sided derivative of $\mu$. The derivative of $\mu$ can also be applied to plant design and the design of robust controllers. Application of the cheap derivative calculation to the design of $\mu$-optimal PID controllers is currently being investigated.

\section{Appendices}

\subsection{Appendix A: Review of Upper and Lower Bounds on $\mu$}

Let us give the well-known upper and lower bounds for $\mu$ with complex uncertainty. First define two subsets of $\mathbf{C}^{n \times n}$

$$
\mathbf{Q}=\left\{Q \in \Delta: Q^{*} Q=I_{n}\right\}
$$

and

$\mathbf{D}=\left\{\operatorname{diag}\left[d_{i} I_{i}\right]: \operatorname{dim}\left(I_{i}\right)=\operatorname{dim}\left(\Delta_{i}\right), d_{i} \in \mathbf{R}, d_{i}>0\right\}$

then it is well-known that

$$
\max _{Q \in \mathbf{Q}} p(Q M) \leq=\mu_{\Delta}(M) \leq \inf _{D \in \mathbb{D}} \bar{\sigma}\left(D M D^{-1}\right) .
$$

A result of Doyle [3] is that the lower bound, $\max \rho(Q M)$, is always equal to $\mu_{\Delta}(M)$. Unfortu-

nately, the maximization is not convex, and comput- ing the global maximum of such functions is, in general, difficult. The lower bound calculation procedure may converge to local maxima. In contrast, the computation of the upper bound is convex. In general though, the upper bound is not equal to $\mu$, though for certain block structures $\Delta$, equality does always hold.

\subsection{Appendix B: Comments regarding Remark 3.3}

$Q$ and $D$ discussed below are defined in Appendix $A$, which reviews the well-known upper and lower bounds on $\mu$.

We defined $M(\alpha)$ by (3.10) instead of (3.12) or (3.13) so that a perturbation in $\alpha$ would result in a near-symmetric perturbation on $M(\alpha)$. Since $Q$ and $D$ are symmetric, it is suspected that using (3.10) for the perturbed $M(\alpha)$ will cause a smaller perturbation in the optimizing $Q$ and $D[20]$.

\section{References}

[1] Balas and Doyle. $\mu$-tools, in preparation, 1991.

[2] Cuffey. TAPPI, 40:190A-197A, 1957.

[3] Doyle. IEE Proc. Part D, 129:242-250, 1982.

[4] Doyle. IFAC Proc., 8:395-402, 1987.

[5] Doyle and Packard. ACC Proc., 3:2147-2152, 1987.

[6] Fan and Tits. TAC, AC-31:734-743, 1986.

[7] Fan, Tits, and Doyle. ACC Proc., 2:1195-1200, 1988.

[8] Freudenberg and Looze. IJC, 35:95-116, 1982.

[9] Kolmogorov and Fomin. Introductory Real Analysis. Dover, 1975.

[10] Laughlin. PhD thesis, Caltech, 1988.

[11] Lundstrom and Skogestad. Comp. Chem. Eng., 4/5:401-413, 1990.

[12] McFarlin. Proc. of 3rd Pulp and Paper Pracess Control Symposium, pages 49-54, 1983.

[13] Morari and Zafiriou. Robust Process Control. Prentice-Hall, Inc., 1989.

[14] Packard. PhD thesis, Berkeley, 1988.

[15] Packard. CDC Proc., 2:1159-1160, 1990.

[16] Pena and Sideris. IJC, 52:753-765, 1990.

[17] Skogestad and Morari. Auto, 29:119-125, 1989.

[18] Smith. PhD thesis, Caltech, 1990.

[19] Young and Doyle. CDC Proc., 3:1230-1235, 1990.

[20] P. M. Young, 1990. personal communication. 